\title{
The Political Turn of Twitch - Understanding Live Chat as an Emergent Political Space
}

\author{
Nadia Ruiz-Bravo \\ University of Gothenburg \\ nadia.ruiz@ait.gu.se
}

\author{
Lisen Selander \\ University of Gothenburg \\ lisen.selander@ait.gu.se
}

\author{
Maryam Roshan \\ University of Gothenburg \\ maryam.roshan@ait.gu.se
}

\begin{abstract}
Research and media have all emphasized the importance of digital platforms such as Twitter and Facebook in contemporary political activism. Yet, little is known of the politicization of other digital platforms, such as Reddit, Twitch, and Discord, and how such politicization is manifested. By politicization, we refer to the emergence of political messages in a "decidedly a-political" space. In this paper, we explore a case of politicization on Twitch, a live streaming platform associated with the gaming community. We focus on the live chat, a central feature of Twitch. Our analysis illustrates rapidly emergent actor roles and their respective use of different features in posting (and objecting to) political messaging. We develop a model that illustrates the dynamic between actors and conclude with implications for research and practice.
\end{abstract}

\section{Introduction}

In recent years, we have witnessed increased public involvement in political conversations on social media platforms [1, 2]. Many studies in the Information Systems (IS) field have analyzed the role that these digital platforms have played in various social movements, collective actions, and political activism around the world $[1,3]$. A large amount of this body of literature has focused on governmental protests (e.g., patriarchy, police) and how the use of different social media platforms (e.g., Facebook and Twitter) enabled or slowed down the advances of these movements [3, 4]. The attention of researchers has largely been on how online movements are shaped, mediated, and sustained. Despite these vibrant and growing research streams, the field has yet to adequately explore the "politicization" of digital platforms as emergent political spaces. We understand politicization as a process where, for a period of time, ideas, messages, facts, etc., are understood as political $[5,6]$. Political spaces are "an area where unconstrained [political] articulation and organization can occur..." [7] (p. 4). Existing research has typically focused on established platforms, such as
Twitter, YouTube, and Facebook-overlooking important developments on other platforms, such as Reddit, Discord, Twitch, and Telegram. These platforms attract large communities of actors, such as the video game community, which are typically peripheral to modern politics but are highly important because of their massive voting power [8]. In this research, we have a particular interest in Twitch, which is one of the world-leading live streaming platforms associated with the video game community.

The video game community constitutes a large and heterogeneous group of people who typically engage and act on multiple digital platforms in parallel [9]. Platforms like Reddit, Twitch, and Discord are emergent arenas for political discussions within the video game community [4, 10]. However, research has largely ignored the video game community platforms as emergent political spaces. Exceptions include game studies that focus on game cultures, hypermasculinity performance in gaming spaces, and technocultures, among many other aspects $[11,12]$. In the IS field, there are some studies on protests/activism in online games $[13,14]$, but only a handful have focused on the politicization of digital platforms [4]. This is unfortunate, as exploring this issue could improve the understanding of how digital platforms shape and reshape the relations and social actions of people existing both inside and outside the technology [15]. Exploring the politicization of digital platforms - and how such politicization is manifested in decidedly "apolitical" communities - can help us to better understand the emergence and scaling of contemporary political activism. In this article, we research an instance of politicization on Twitch by focusing on one of its most significant features: the live chat. We ask the following question: How do roles and associated feature usage emerge and manifest in the politicization of live chats on Twitch?

To answer this question, we traced signs of politicization in live chats during multiple e-sports events on Twitch. The political issue at hand concerned Blizzard, a world-leading gaming company, and its 
associations to the Hong Kong movement in 2019. Our research contributes to the growing literature on political activism on digital platforms and to the chatroom literature.

\section{Literature Review}

\subsection{Digital platforms as political spaces}

A digital platform is a digital environment where digital resources, like software, get to function, allowing for communication between different actors [16, 17]. The literature on digital platforms is vast and contains a wide range of approaches, including the study of its architecture [18], application development [19, 20], digital content [21], political activism, digital collective action, and social movements [1,3].

Research on digital platforms as political spaces has explored different actors' roles and behaviors and the affordances each platform has to offer [2, 22]. For example, in a recent study on the Bersih movement in Malaysia, Leong et al. [23] showed the importance of Facebook in formalizing different roles of movement participants over time. They revealed the emergence of political Facebook groups that shared a similar cause. Others (see [3, 24]) have also demonstrated the importance of political group formation on Facebook. In another important study, Vaast et al. [2] studied Twitter and identified the emergence of three interdependent roles during the Gulf of Mexico oil spill: advocates, supporters, and amplifiers. Advocates were "initiating the activity by advocating for the cause (...) and encouraging others to participate" (p. 1192). Supporters were participating and supporting the cause by qualifying the activism. Amplifiers were sharing the content created by the Advocates and the Supporters. These actors used the affordances of Twitter (e.g., direct messages ("@"), hashtags ("\#”), and retweets) to their advantage to make the cause they were advocating more visible. Among the research done on other platforms, such as Reddit, as a political space, Guimaraes et al. [22] proposed four types of conversations related to political discussions: harmony, discrepancy, disruption, and dispute. Harmony involves discussions that are in agreement or without polarized debates. Discrepancy is characterized by a certain amount of negative or mixed responses. Disruption occurs in conversations that shift in terms or reactions to the discussion. Dispute represents the arguments or disagreements over a given topic that could have polarized opinions. However, none of these studies have looked into public live chats as a political space. Moreover, the above-mentioned studies provide insights into political organizing and manifestations rather than the transformation of a decidedly a-political space into a political space.

\subsection{Political activism in the video game community}

The video game community is rarely involved in international politics. Members of this community typically consider themselves as a-political $[25,26]$. For example, early in 2020, the CEO of the company Epic Games said, "we as companies need to divorce ourselves from politics [...] we as platforms should be neutral" (Tim Sweeney speech at DICE 2020 [27]). Yet, while the prevailing norm has been to stay out of politics, historically, there have been different political agendas/themes/ideologies that have been part of the industry and their platforms $[28,29]$. Such politics are manifested in war games, strategic games, or fantasy/dystopian games, among many others [30].

Virtual protests and manifestations on different video game platforms have increased in the midst of the COVID-19 pandemic [31]. For example, the game Animal Crossing gained attention in 2020 for being one of the most used games for virtual protests [32]; other games (mostly online games) have also been protagonists of such political manifestations in previous years (e.g., League of Legends and World of Warcraft) $[13,14]$. Even so, to the best of our knowledge, there is little if any research focusing on the politicization of live chats.

\subsection{Twitch}

Twitch is a live video streaming platform owned by Amazon and operated by Twitch Interactive [33]. This platform had approximately 9.36 million active streamers in April 2021 [34] and 3.75 million concurrent viewers on average in June 2021 [35]. Among the many features and characteristics of Twitch, the embedded chat function that is displayed and streamed along with the live video is one of the unique traits of this platform $[36,37]$. In the chat, viewers of the stream can interact with the streamer (the person doing the stream), creating a sense of closeness that other platforms do not have or are still exploring $[36,37]$. The behavior in the chat can vary depending on the type of the streamer and channel, but in one of the most viewed streaming channels - esports - broadcasts are often chaotic and fast-paced, making them a strange experience for a person who is new to e-sports [36, 37]. This chaotic behavior is known as chat spamming or merely spamming. Spamming refers to massive messaging of a particular phrase, emote, or word in the chat [38]. Contrary to regular spam, which has malicious intentions, chat spam is simply a mindless action people engage in to have fun in the chat while watching a stream, similar to the way fans shout and cheer in a physical stadium [39, 40]. Messages in the chat, either spam or not, tend to be 
accompanied by emotes. Emotes are "Twitch-specific emoticons that viewers and streamers use to express a number of feelings in [the] chat" [41]. Examples of emotes include the following: 2,0 ,

In the chat, there are other actors besides the stream's viewers, such as moderators. "Moderators (also known as mods) ensure that the chat meets the behavior and content standards set by the broadcaster by removing offensive posts and spams that detract from conversations" [42]. The moderators' role is essential for the streamers, especially for big video game companies that broadcast e-sports, as the mods ensure that good practices are maintained in the chat.

Twitch has been primarily used by both video game players and video game companies (big and small) to live-stream recreational video gaming and broadcast esports championships. Increasingly, however, people are turning to Twitch to engage in political conversations and actions. The New York Times covered some of these occurrences and reported that Twitch has "transformed into an unexpected hub of social activism" [43]. Politicians from all over the world (many from the USA, Sweden, Russia), and even governmental institutions (mostly from Englishspeaking countries but also from all over the world), had found in this platform a way to reach young voters [44, 45]. This shows a growing interest in this platform as a political space, and increasingly its users (mostly video gamers) are the target of this interest.

\subsection{Chatrooms}

The literature related to chats dates back almost twenty years. A chatroom, or Internet Relay Chat (IRC), is a digital environment where people can have (text) conversations in real-time (synchronous) with others [46]. Research on chatrooms has explored different characteristics, such as the type of people that certain chatrooms attract, the topics discussed in them (similar interests), and the strategies adopted by the chatroom participants [46]. Chats, according to Greenfield and Subrahmanyam [46], are "an amalgam of spoken and written language" (p. 722), where the conversation is typed on a keyboard and read on a screen (written language), but in a short, incomplete, and often grammatically simple form, similar to spoken language. Brevity in texts is what enables chats to resemble realtime (oral) conversations. Online conversations, according to the authors, "typically have (1) several topics being discussed in parallel by partly overlapping groups of people, (2) many turns between an utterance and its response, (3) people contributing to several conversations, and (4) relatively quick topic decayi.e., relatively short conversations on a given topic" [46] (p. 716). Greenfield and Subrahmanyam discussed the strategies that participants adopt in the chats: repetition, vocative cues, visual cues, and conventionalized chat codes [46]. "Repetition is (...) frequently used by chat participants to identify relevant utterances" (p. 728). Vocative cues are used when chat participants include the names of other chat participants in the message. Visual cues are used to identify chat participants (e.g., colors, numbers on nicknames). Conventionalized chat codes are "a group of conventions or chat codes that have been constructed (and co-constructed) as specific adaptations to the chat situation" [46] (p. 732).

More recent research on chats has explored e-sports streaming and the use of different features, such as emojis and other graphical content, as well as communities [36, 37].

Ford et al. [37] proposed three interrelated practices of communication in live chats: "shorthanding", bricolage, and voice-taking. Shorthanding "is the contraction of text into a smaller space" (p. 863). It "occurred in the form of acronyms, abbreviations, emotes, and single word commands" (p. 864). Bricolage is "the practice of recombining a small set of resources at hand (such as known characters, tropes, and images) to construct collective narratives" (p. 865). Bricolage on Twitch can manifest as "recombining emotes, stock phrases (...), and copypastas" (p. 865). Copypasta is "the practice (...) where people cut and paste anecdotes and fiction from around the web, mixing and remixing them without citation or explicit links to the original" (Chess \& Newsom cited in [47] (p.162)). "Voice-taking is the adoption of shared viewpoints, perspectives, or mannerisms" [37] (p. 863).

\section{Method}

Our study is centered around the usage of the live chat feature on Twitch. We followed a live stream esports tournament of the game Hearthstone ("PlayHearthstone" on Twitch) through 25 different chatlogs. We traced the emergence of actor roles, their behavior, and usage of different chat features as participants started to propose political messages related to the protests in Hong Kong in 2019.

\subsection{Case background}

On October $6^{\text {th }}, 2019$, during a Grandmasters AsiaPacific e-sports championship, the winner of the round, known as Blitzchung, voiced his support for the Hong Kong protests. He expressed his concerns in an aftertournament interview streamed on Twitch. Blizzard, the game creator and host of the event, punished the player by banning him from the current tournament, removing his prize money (around USD 4000), and prohibiting 
him from playing in another Grandmaster tournament for a year. The casters (interviewers) were also punished by Blizzard by being fired. Blizzard said the punishment was because the player and the casters broke the rules of the tournament by bringing political discussions, offending the public, and/or causing damage to Blizzard's brand [48]. The punishments triggered a wave of backlash from the gamer community, which led to boycott campaigns, loss of sponsors, physical protests, and even letters from the US Congress aimed at pressuring Blizzard to reconsider the punishment decision [49, 50, 51]. Gamer protestors took over different digital platforms to bring the conversation about the situation in Hong Kong to the gamer community. On Twitch, where the Grandmaster championship was still being played, protestors flooded the chat in every live stream of the official Hearthstone channel to protest. These protests were at the center of our analysis.

After all the protests, Blizzard decided to reduce the punishment for the player, returning the prize money and lowering the ban to six months, and for the casters, rehiring them but with a six-month ban as well. Nonetheless, this did not diminish the heated sentiments, and protests went on for weeks.

\subsection{Data collection}

We first read about the Blizzard events in a New York Times article published in October 2019 [52]. It immediately caught our attention as an interesting case of politicization of the gaming community. We engaged in developing Python scripts to scrape the different chat rooms in Twitch. We collected 25 chatlogs of 25 video streaming events (from October $9^{\text {th }}$ to December $1^{\text {st }}$, 2019). This included 24 Hearthstone championship broadcasts. We also downloaded a broadcast of a global Blizzard conference (BlizzCon), where we anticipated that we would get additional information about the protests. We gathered 342,600 messages in the 25 live chats. The broadcasts were chosen because they followed immediately after the Blitzchung interview.

\subsection{Data analysis}

We followed the development of the protest over time and performed thematic analysis. Our study was exploratory, and we continuously iterated between data analysis and theory. The data analysis involved reading and familiarizing ourselves with the data. This work involved preparing the data in Excel and converting it to the compatible format admitted by Atlas.ti (a qualitative analysis software program), which was the program we used to code our data. We started by analyzing the first chatlog. We instantly noticed a high level of redundancy in the data set (e.g., spams). For instance, in the first chatlog, we saw that around $30 \%$ of the messages were spam. This proportion was also valid for the remaining 24 chatlogs. With this awareness, we engaged in manually coding the remaining dataset. Importantly, the messages were short in length, thus relatively quickly coded.

We recognized different types of messages that represented various types of behavior. The most salient messages in our data, and the first ones that we encountered, were messages about mobilizing protests in Hong Kong and criticizing Blizzard. We noticed how different features were used to express such mobilization and protest and how different roles emerged in the data set. We turned to the literature on political activism, especially Vaast et al. [2], to better understand the different roles. We quickly realized, however, that the live chats on Twitch have features that allowed for new forms of actor engagement as well as the emergence of new roles. We then turned to the literature on chatrooms to complement our understanding of what we saw in the data. We found the work of Ford et al. [37] to be particularly relevant. Ultimately, we referred to this group of actors, and their respective feature use, as "mobilizers." We also noticed the emergence of "opponents" in the chatroom. These were actors who were opposing the mobilization against Blizzard. We noticed that they mimicked the mobilizers. For example, if the mobilizers would write "free Hong Kong," the opposers would reply with "free King Kong." We were intrigued by this finding. Although less dominant in the chatlogs, the "opponents" were visible throughout all chatlogs. Finally, we also found that more "peripheral" actors were part of the politicization process. These actors included moderators and (esports) spectators (including bots). These last actors were a minority but their actions, mostly the moderators, were crucial for the development of the events in the chat. Next, we detail these roles as we present our results.

\section{Results}

The analysis of the data revealed four groups of actors that fueled the politicization process: Mobilization, Opposition, Moderators, and the Spectators group. In the following sections, the characteristics of these groups will be explained in detail.

\subsection{Mobilization group}

To a large degree, it was the Mobilization group that started to transform Twitch's chatroom into a protest space. Their participation was the most salient in 
the dataset. We identified two types of actors in this group that we refer to as Spammers and Awareness Creators.

4.1.1. Spammers. These actors dominated the chat by repeating political messages and using features such as emojis and emotes. The spamming typically included political slogans. The most spammed phrase was "Free Hong Kong, the revolution of our times," which was a quote from the interview with the e-sports player that led to his punishment by Blizzard (this slogan was an insitu slogan used by Hongkongers during the protests in 2019 [53]). The Spammers made extensive use of emojis and emotes in the spam messages.

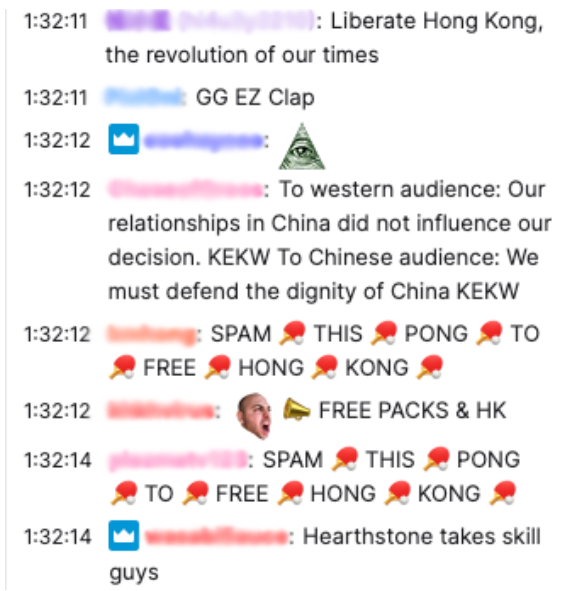

Figure 1. Example of the live chat on Twitch during one of the Hearthstone championships in October 2019

Spamming, as such, was not new to the live chats; rather, it was the political content of the spam that was transformative and elicited reactions.

4.1.2. Awareness Creators. This second set of actors in the Mobilization group focused on bringing more context to the conversation around Hong Kong and why they considered it important to protest against Blizzard. Their messages were politically charged and more extensive than those of the Spammers. An example message was, "Chinese government should not be allowed to suppress free speech abroad with the help of American companies." They, too, often included emojis and emotes, accentuating the sentiment of the messages (see examples in Table 1). The Awareness Creators sometimes re-used the slogans of the Spammers, but they were not used as spam but rather to give an opinion or a historical fact about Hong Kong's situation, for example, "FREE HONGKONG, FREE MAKAO, FREE TIBET, TAIWAN NO.1" (see more examples in Table 1). The political messages of the Awareness Creators and the Spammers triggered several reactions from the Opposition group.

Table 1. Examples of messages of the Mobilization group

\begin{tabular}{|c|c|}
\hline Actors & Data examples \\
\hline Spammers & $\begin{array}{l}\text { - "Free Hong Kong! Revolution of } \\
\text { our times!" } \\
\text { - "Blizzard banning chat E FREE } \\
\text { - "APEECH C" } \\
\text { - "\#FreeHongKong }\end{array}$ \\
\hline $\begin{array}{l}\text { Awareness } \\
\text { Creators }\end{array}$ & $\begin{array}{l}\text { - "This has blown up in Blizzard's } \\
\text { face. Even congressmen and } \\
\text { women are saying Blizzard } \\
\text { shouldn't pander to communist } \\
\text { governments." } \\
\text { " "... } 9 . \cdot \text { BOYCOTT } \\
\text { BLIZZARD "Blizzard is a company that will } \\
\text { trade civil rights for money, don't } \\
\text { let them silence us" }\end{array}$ \\
\hline
\end{tabular}

\subsection{Opposition group}

In the analysis of the data, a growing number of messages appeared countering the arguments of the Mobilization group. These messages, although less dominant than the Mobilization group, expressed frustration and dissatisfaction with the sudden politicization of the live chat, criticism about the nature of the activism, and even mockery. We refer to this group as Opposition. We identified two distinct actor roles in this group that we refer to as Mimics and Critics.

4.2.1. Mimics. These actors, as the name suggests, focused on mimicking the actions and messages of the Spammers. The Mimics spammed almost as much as the Spammers, which caused noise and distraction in the live chats. By distorting the phrases and slogans of the Spammers, the Mimics created confusion and discredited the messages of the Mobilization group. For example, the message "Free King Kong, the evolution of our times" was developed and frequently used by the Mimics. Another example was "FREE DONKEY KONG HE DID NOTHING WRONG." Their messages were replicated vastly, and as mentioned above, added to the distortion and "noise" in the live chats (see more examples in Table 2).

4.2.2. Critics. Contrary to the Mimics, the Critics did not distort messages or spam the chat. Rather, they responded directly to the Mobilization group. The messages expressed by these actors were heterogeneous and involved both discontent for the politicization of the chatroom and engagement in the political conversation. 
Examples include "Blizzard did nothing wrong, they just enforced a rule that was broken" and "keep politics out of gaming, I watch this stream to get away from politics" (see more examples in Table 2).

Table 2. Examples of messages of the Opposition group

\begin{tabular}{|c|c|}
\hline Actors & Data examples \\
\hline Mimics & 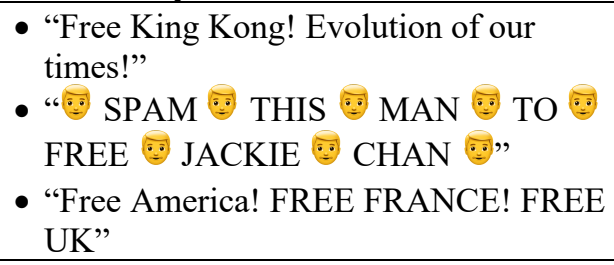 \\
\hline Critics & $\begin{array}{l}\text { - "Support Blizzard. China's not our } \\
\text { concern" } \\
\text { - "@ We're here for video } \\
\text { games, not your politics. Typing words } \\
\text { does nothing for anyone. Also get your } \\
\text { caps lock fixed" } \\
\text { - "Most of these wannabe activists have } \\
\text { the mental age of a 15-year-old, they } \\
\text { don't comprehend the complexity of this } \\
\text { world and seek shelter in simplistic } \\
\text { answers to complex questions." } \\
\text { - "Free Catalonia!... No? Free Chile!... } \\
\text { No? Not trendy huh" }\end{array}$ \\
\hline
\end{tabular}

\subsection{Moderators}

Twitch, being a (digital) public space, has rules and regulations for participation. The Moderators on Twitch act as keepers of these rules. The Moderators' job is to keep an eye on the live chat and act accordingly to ensure that everyone is following the rules imposed by the streamer, in this case, Blizzard.

Initially, the Moderators were not active in the live chats but were called upon by the Opposition group, who was particularly annoyed by the spamming, as shown in the following example: "WHY DONT YOU BAN THE SPAMMERS" (see more examples of this in Table 3). Slowly, the Moderators started to take some actions, like eliminating political messages and banning people for 24 hours. However, these practices only triggered the posting of more political messages. The following example is from the Mobilization group: "this chat is highly censored like China" (see more examples in Table 3). With the rapidly growing number of political messages, the Moderators started to explicitly warn people that if their actions continued, there would be consequences (see examples in Table 3). This further triggered the posting of more political messages, for example, "Are you banning people for saying Hong Kong now? Really? So fascist Blizzard" or "WHY THE
FRIK DOES IT CENSOR ME EVERY TIME I SAY SOMETHING. I SAID HI AND IT CENSORED IT."

Table 3. Examples of messages the Moderators got or made

\begin{tabular}{|c|c|}
\hline Actors & Data examples \\
\hline $\begin{array}{l}\text { Reactions } \\
\text { from the } \\
\text { Critics }\end{array}$ & $\begin{array}{l}\text { - "BAN ALL PROTESTERS " } \\
\text { - "Can u chat about the game } \\
\text { instead of politics, what } \mathrm{r} \text { MODS } \\
\text { doing" }\end{array}$ \\
\hline $\begin{array}{l}\text { Moderators' } \\
\text { voice }\end{array}$ & $\begin{array}{l}\text { - "Please don't fill up the entire } \\
\text { chat, @ (warning)" } \\
\text { - "Calm the spam, @) } \\
\text { (warning)" } \\
\text { - "Calm it with those symbols, } \\
\text { @ (warning)" }\end{array}$ \\
\hline $\begin{array}{l}\text { Reactions } \\
\text { from the } \\
\text { Awareness } \\
\text { Creators }\end{array}$ & $\begin{array}{l}\text { - "So will we get banned for } \\
\text { mentioning China and how we } \\
\text { need to free Hong Kong" } \\
\text { - "Haha I can't believe bliz would } \\
\text { censor the term CCP. Must be } \\
\text { scared of big bad China!" }\end{array}$ \\
\hline
\end{tabular}

\subsection{Spectators group}

The data showed a fourth group of users who seemed to be in the margins of the conflict between the Mobilization group and the Opposition group. We call this group Spectators. This group includes both human users and bots. The Spectators group did not engage in any of the conversations or interactions of the Mobilization and Opposition group. Yet, they were explicit about their presence in observing the developments in the live chat or receiving reactions from other users. We identified two actor roles in this group: Bystanders and Bots.

4.3.1. Bystanders. These actors were active spectators of the discussion between the Mobilization group and the Opposition group. They did not take any "side" in the discussion; rather, they seemed to enjoy the interactions between the two main groups (e.g., "This is gonna be so fun in Blizzcon: Table 4).

4.3.3. Bots. These actors were the only non-human actors we could identify in our data. They were clearly named as bots, so they were easy to identify (e.g., Moobot). Their messages varied from giving information about the particular brand they represented (e.g., Tespa) to encouraging participants of the chat to ask questions to the casters, such as "Do you have a funny or interesting question that you would like to ask our casters?Tweet at@TeamTespa and ifyour question is read on stream, you will earn the Dalaran Flame 
Card Back!" (See more examples in Table 4). Although the Bots did not engage in the discussion, they received varied reactions from other actors (e.g., Awareness Creators) who associated the bots with Blizzard. Like the following message that followed a question made by one bot, "@TeamTespa how do you feel about working for a company that supports oppression?", or "yeah, I have a question... who hired these mods?"

Table 4. Examples of messages of the Spectators group

\begin{tabular}{|l|l|}
\hline Actors & Data examples \\
\hline Bystanders & - "a it's going to be a \\
& fiesta "I don't even care about TESPA. \\
& I came here for the comments" \\
& - "Mods will have fun day \\
\hline Bots & "Tespa believes in a world \\
& where gaming is celebrated by \\
& people of all ages, recognized as \\
& a force for good, and a catalyst \\
& for bringing people together. \\
& Learn more about Tespa here: \\
& https://tespa.org/about" \\
& "Hearthstone Global Games \\
& bracket can be found here: \\
& https://playhearthstone.com/espo \\
rts/tournament/7210"
\end{tabular}

\section{Discussion and implications}

Our study offers three interconnected theoretical contributions.

First, we contribute to the literature on the politicization of digital spaces, particularly the case of live chats on video game-related platforms. The existing literature has widely addressed the usage of social media platforms, such as Facebook and Twitter, for political messages [2, 3]. However, these platforms have fundamentally different characteristics than a live chat on a streaming platform like Twitch.

On Twitch, participants gather to follow particular events and engage through live chats [38]. The live chat function is public and open. In this sense, Twitch live chats foster opinion heterogeneity (diversity of voices in a social network space) rather than echo chambers [54]. The practices and strategies for coherent communication suggested by Ford et al. [37] (shorthanding, bricolage, and voice-taking) and Greenfield and Subrahmanyam [46] (repetition, vocative cues, visual cues, and conventionalized chat codes) were present in our study. We noticed the use of emoticons and emotes (visual cues) as well as the vocative cues of the Spammers. The emergence of different roles, as identified in our case, is a result of such diversity and heterogeneity in interests/political views.

We extend the existing literature by identifying the roles that emerge in the politicization of a video game streaming live chat - a digital space that is decidedly apolitical [26]. We found interesting similarities with the different roles identified on Twitter by Vaast et al. [2]. The Advocates, Supporters, and Amplifiers identified by Vaast et al. [66] share similarities with what we refer to as Spammers and Awareness Creators on Twitch. The main activity of these groups was, in a way, to advocate, support, and amplify the cause's message; however, the delivery of such activities and their reach was different in the live chat than on Twitter. Additionally, we identified the presence of additional roles (Opposition, Moderators, and Spectators) in the live chat context. In the Opposition group, we recognized similarities between the Mimics behavior and the internet troll behavior identified by Phillips [55]. Mimics, like trolls, engaged in derailing the conversation and distorting the original messages (of the Mobilization group in this case). However, while the trolls tend to be more offensive, aggressive, and mischievous [55], the Mimics seemed less harmful and more playful. We acknowledge that the Mimics could be an instantiation of trolls as, similar to trolls, they are always looking for the inconsequential laughter in the situations often referred to as "lulz" [55]. For the Moderators, there are some studies that include Moderators (or their activity), but the setups of these studies are quite different from our study $[38,56]$. Research on Moderators on Twitch has not studied them in politicized environments [38], and the investigation has focused on a more political arena, not live chats [56]. Additionally, there is relatively little research on the dynamic relationship between Opposers and Proposers (Mobilizers) online [2, 3]. The noticeable presence of Opposers in our case could also be traced to the heterogeneity of the chatroom crowd. The efforts of the Opposition group (and Moderators) somewhat resembled the role of a countermovement [54, 57]. The actions taken by both the Opposition group (especially the Critics) and the Moderators responded with the prevailing norms of the video game industry to keep the community a-political [27].

We propose a dynamic model (Figure 2) that illustrates the process of politicization of live chats. Previous literature has addressed the politicization of different digital platforms. Our model extends this stream of literature by illustrating the dynamics of interactions that take place among the emerging roles in the politicization process. 


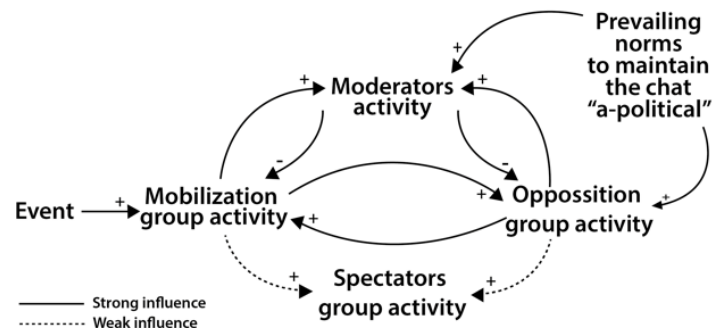

Figure 2. Model of the dynamic between actor roles in the live chat on Twitch

The model describes how external events can trigger a politicization process in a live chat. We see how the different actor groups influenced and reinforced each other and how the Moderators tried to push back on this spiraling loop. Triggered by the political events, the Mobilization group start to politicize the 'decidedly' a-political live chats by posting political messages. The actors in the Opposition group react to the messages to stop the politicization. However, this acting and reacting create a reinforcing loop among the Mobilization and Opposition groups. When the Moderators get involved, they act through controlling mechanisms to push back on both groups and return the chat space to being apolitical. Both the Mobilization and Opposition groups react to the Moderator's intervention. These dynamics influence the peripheral activity of the Spectators who do not directly engage but acknowledge the situation.

\section{Limitations and future research}

Our contributions need to be considered in light of the limitations of this research. First, our findings are based on a single case study, which might affect their generalizability. We were also tied to the limitation of scraping tools to collect our data. In addition, since our data is secondary, we have not talked to the users of the live chat to understand the psychological traits in their actions and behavior. Lastly, we recognize that the analysis of this case study applies mostly to large and public outlets such as e-sports. We cannot generalize our findings to smaller and/or private streams.

Given these limitations, we encourage future researchers to examine the roles discussed in this paper to see if they appear in other contexts of emergent politicization of platforms that have a live chat feature such as Facebook Live, YouTube Live, or Instagram Live. Future research could explore the action repertoires used in politicized live chats and study esports chat spaces in search of more instances of political activism.

\section{Conclusion}

In this paper, we sought to direct attention to underexplored digital platforms (e.g., Twitch) in the context of contemporary political activism. We were particularly interested in the gaming community, as it is a huge group with substantial potential political power. We identified the rapid formation of user groups and the emergence of politicization in the chatrooms on the Twitch platform. We suggest that the IS field should broaden its attention to include other (less publicly scrutinized) digital platforms as potential political spaces of significance. Focusing on other digital platforms and keeping an open mind to the associated (digital) cultures will be critical for understanding future political activism.

\section{References}

[1] Selander, L., and S.L. Jarvenpaa, "Digital Action Repertoires and Transforming a Social Movement Organization”, MIS Quarterly 40(2), 2016, pp. 331-352.

[2] Vaast, E., H. Safadi, University of Georgia, L. Lapointe, McGill University, and B. Negoita, "Social Media Affordances for Connective Action: An Examination of Microblogging Use During the Gulf of Mexico Oil Spill”, MIS Quarterly 41(4), 2017, pp. 1179-1205.

[3] Stewart, M., and U. Schultze, "Producing solidarity in social media activism: The case of My Stealthy Freedom", Information and Organization 29(3), 2019, pp. 100251.

[4] Buyukozturk, B., S. Gaulden, and B. Dowd-Arrow, "Contestation on Reddit, Gamergate, and movement barriers", Social Movement Studies 17(5), 2018, pp. 592-609.

[5] Palonen, K., "Four Times of Politics: Policy, Polity, Politicking, and Politicization", Alternatives: Global, Local, Political 28(2), 2003, pp. 171-186.

[6] Wiesner, C., ed., Rethinking politicisation in politics, sociology and international relations, Palgrave Macmillan, Cham, Switzerland, 2021.

[7] Tkacheva, O., Internet freedom and political space, RAND National Research Defense Institute, Santa Monica, CA, 2013.

[8] Smith, N., "The gamer vote: Democrats lean into video games to aid Biden campaign”, Washington Post, 2020. https://www.washingtonpost.com/videogames/2020/10/22/video-games-2020-presidentialelection-biden-trump/

[9] Won Jung, C., 'Role of gamers' communicative ecology on game community involvement and self-identification of gamer", Computers in Human Behavior 104, 2020, pp. 106164.

[10] Hawking, T., "How a 57-hour Donkey Kong game struck a blow against online toxicity", the Guardian, 2019. http://www.theguardian.com/games/2019/jan/22/howa-57-hour-donkey-kong-twitch-stream-struck-a-blowagainst-gamergate 
[11] Chess, S., and A. Shaw, "A Conspiracy of Fishes, or, How We Learned to Stop Worrying About \#GamerGate and Embrace Hegemonic Masculinity", Journal of Broadcasting \& Electronic Media 59(1), 2015, pp. 208220.

[12] Mortensen, T.E., "Anger, Fear, and Games: The Long Event of \#GamerGate", Games and Culture 13(8), 2018, pp. 787-806.

[13] McKenna, B., "Creating convivial affordances: A study of virtual world social movements", Information Systems Journal 30(1), 2020, pp. 185-214.

[14] Stokes, B., and D. Williams, "Gamers Who Protest: Small-Group Play and Social Resources for Civic Action", Games and Culture 13(4), 2018, pp. 327-348.

[15] Baskerville, R.L., M.D. Myers, and Y. Yoo, "Digital First: The Ontological Reversal and New Challenges for IS Research", 2019, pp. 35.

[16] Constantinides, P., O. Henfridsson, and G.G. Parker, "Introduction-Platforms and Infrastructures in the Digital Age", Information Systems Research 29(2), 2018, pp. 381-400.

[17] Tiwana, A., B. Konsynski, and A.A. Bush, "Research Commentary -Platform Evolution: Coevolution of Platform Architecture, Governance, and Environmental Dynamics", Information Systems Research 21(4), 2010, pp. 675-687.

[18] Yoo, Y., O. Henfridsson, and K. Lyytinen, "Research Commentary - The New Organizing Logic of Digital Innovation: An Agenda for Information Systems Research", Information Systems Research 21(4), 2010, pp. 724-735.

[19] Eaton, B., S. Elaluf-Calderwood, London School of Economics and Political Science, et al., "Distributed Tuning of Boundary Resources: The Case of Apple's iOS Service System”, MIS Quarterly 39(1), 2015, pp. 217-243.

[20] Ghazawneh, A., and O. Henfridsson, "Balancing platform control and external contribution in third-party development: the boundary resources model: Control and contribution in third-party development", Information Systems Journal 23(2), 2013, pp. 173-192.

[21] Nylén, D., and J. Holmström, "Digital innovation in context: Exploring serendipitous and unbounded digital innovation at the church of Sweden", Information Technology \& People 32(3), 2019, pp. 696-714.

[22] Guimaraes, A., O. Balalau, E. Terolli, and G. Weikum, "Analyzing the Traits and Anomalies of Political Discussions on Reddit”, pp. 9.

[23] Leong, C., I. Faik, F.T.C. Tan, B. Tan, and Y.H. Khoo, "Digital organizing of a global social movement: From connective to collective action", Information and Organization 30(4), 2020, pp. 100324.

[24] Kavada, A., "Creating the collective: social media, the Occupy Movement and its constitution as a collective actor", Information, Communication \& Society 18(8), 2015, pp. 872-886.

[25] Bown, A., "Video games are political. Here's how they can be progressive", the Guardian, 2018. http://www.theguardian.com/games/2018/aug/13/video -games-are-political-heres-how-they-can-beprogressive
[26] Campbell, C., "Why are game companies so afraid of the politics in their games?", Polygon, 2018. https://www.polygon.com/2018/6/20/17480666/videogames-companies-lying-politics

[27] IGN, Tim Sweeney's Full DICE 2020 Talk, 2020.

[28] Parkin, S., "The Division 2 and the Severing of Politics from Video Games", The New Yorker, 2019. https://www.newyorker.com/science/elements/thedivision-2-and-the-severing-of-politics-from-videogames

[29] Sisler, V., "Videogames and Politics", Entermultimediale 2, Centre for Independent Culture Palace Akropolis and CIANT - International Centre for Art and New Technologies (2005), 38-40.

[30] Pfister, E., “„Keep your Politics out of my Games!“””, Spiel-Kultur-Wissenschaft, https://spielkult.hypotheses.org/1566

[31] Schofield, D., "Black Lives Matter meets Animal Crossing: how protesters take their activism into video games", the Guardian, 2020. http://www.theguardian.com/games/2020/aug/07/black -lives-matter-meets-animal-crossing-how-protesterstake-their-activism-into-video-games

[32] Tidmarsh, B., "Holding Protest Posters And Handheld Consoles: Activism In Animal Crossing: New Horizons", 2021. https://ir.library.illinoisstate.edu/urs2021eng/1

[33] Kim, E., “Amazon Buys Twitch For $\$ 970$ Million In Cash”, Business Insider. https://www.businessinsider.com/amazon-buys-twitch2014-8

[34] Clement, J., "Global active streamers on Twitch 2021", Statista, 2021. https://www.statista.com/statistics/746173/monthlyactive-streamers-on-twitch/

[35] TwitchTracker, "Twitch Viewers Statistics", TwitchTracker, 2021. https://twitchtracker.com/statistics/viewers

[36] Diwanji, V., A. Reed, A. Ferchaud, J. Seibert, V. Weinbrecht, and N. Sellers, "Don't just watch, join in: Exploring information behavior and copresence on Twitch", Computers in Human Behavior 105, 2020, pp. 106221.

[37] Ford, C., D. Gardner, L.E. Horgan, et al., "Chat Speed OP PogChamp: Practices of Coherence in Massive Twitch Chat", Proceedings of the 2017 CHI Conference Extended Abstracts on Human Factors in Computing Systems, ACM (2017), 858-871.

[38] Seering, J., R. Kraut, and L. Dabbish, "Shaping Pro and Anti-Social Behavior on Twitch Through Moderation and Example-Setting", Proceedings of the 2017 ACM Conference on Computer Supported Cooperative Work and Social Computing, ACM (2017), 111-125.

[39] Chow, E., "Faculty of Philosophy ICS-Programme", pp. 107.

[40] Poyane, R., "Toxic Communication on Twitch.tv. Effect of a Streamer", Digital Transformation and Global Society, Springer International Publishing (2019), 414 421. 
[41]

Twitch,

"Emotes",

2021. https://www.twitch.tv/creatorcamp/en/learn-thebasics/emotes/

[42] Twitch, "Guide to Building a Moderation Team", 2021. https://help.twitch.tv/s/article/guide-to-building-amoderation-team?language $=$ en_US

[43] Lorenz, T., "How Hasan Piker Took Over Twitch", The New York Times, 2020 https://www.nytimes.com/2020/11/10/style/hasanpiker-twitch.html

[44] Basu, T., "AOC's Among Us livestream hints at Twitch's political power", MIT Technology Review, 2020. https://www.technologyreview.com/2020/10/21/10110 38/aocs-among-us-livestream-hints-at-twitchs-politicalpower/

[45] Foxall, S., "Swedish politician uses his own Hearthstone stream to explain eSports legislation", PCGamesN, 2017.

https://www.pcgamesn.com/hearthstone/hearthstonesweden-parliament-twitch-stream

[46] Greenfield, P.M., and K. Subrahmanyam, "Online discourse in a teen chatroom: New codes and new modes of coherence in a visual medium", Journal of Applied Developmental Psychology 24(6), 2003, pp. 713-738.

[47] Ondrak, J., "Spectres des Monstres: Postpostmodernisms, hauntology and creepypasta narratives as digital fiction", Horror Studies 9(2), 2018, pp. 161178.

[48] Blizzard Entertainment, "Hearthstone Grandmasters Asia-Pacific Ruling”, 2019. https://playhearthstone.com/en-us/blog/23179289/

[49] Chalk, A., "Bipartisan members of congress call on Blizzard to reverse Blitzchung punishment", PC Gamer, 2019. https://www.pcgamer.com/bipartisan-membersof-congress-call-on-blizzard-to-reverse-blitzchungpunishment/
[50] Kelly, M., “After Hearthstone player's ban, Blizzard is in hot water with lawmakers", The Verge, 2019. https://www.theverge.com/2019/10/8/20905181/blizzar d-hearthstone-player-ban-marco-rubio-ron-wydenchina-hong-kong-protests-blitzchung

[51] Wyden, R., M. Rubio, A. Ocasio-Cortez, M. Gallagher, and T. Mailnowski, "Wyden Letter to ActivisionBlizzard on Hong Kong protest-related ban 2019-1018 ", 2019. https://upload.wikimedia.org/wikipedia/commons/6/63/ Wyden_Letter to ActivisionBlizzard_on_Hong_Kong_protestrelated ban_20191018.pdf

[52] Allen Clark, P., "What to Know About Blizzard, Hong Kong and the Controversy Over Politics in Esports", Time, 2019. https://time.com/5702971/blizzard-esportshearthstone-hong-kong-protests-backlash-blitzchung/

[53] Davidson, H., "'Liberate Hong Kong' slogan banned as protesters lie low", the Guardian, 2020. http://www.theguardian.com/world/2020/jul/02/hongkong-protesters-laying-low-following-mass-arrestschina

[54] Guidetti, M., N. Cavazza, and A.R. Graziani, "Perceived Disagreement and Heterogeneity in Social Networks: Distinct Effects on Political Participation", The Journal of Social Psychology 156(2), 2016, pp. 222-242.

[55] Phillips, W., "Meet the trolls", Index on Censorship 40(2), 2011, pp. 68-76.

[56] Selander, L., and S.L. Jarvenpaa, "Xenografting in Political Activism: Co-Existing Logics Powered by Resource Injections", Academy of Management Discoveries, 2020, pp. amd.2019.0052.

[57] Mundt, M., K. Ross, and C.M. Burnett, "Scaling Social Movements Through Social Media: The Case of Black Lives Matter", Social Media + Society 4(4), 2018, pp. 205630511880791. 